\title{
EMG Based Control of a Robotic Exoskeleton for Shoulder and Elbow Motion Assist
}

\author{
Mohammad H. Rahman, Cristobal Ochoa-Luna, and Maarouf Saad \\ École de Technologie Superieure, Montreal, Canada \\ Email: mohammad.rahman3@mail.mcgill.ca, cristobal.ochoa-luna.1@ens.etsmtl.ca, maarouf.saad@etsmtl.ca \\ Philippe Archambault \\ McGill University, Montreal, Canada \\ Email: philippe.archambault@mcgill.ca
}

\begin{abstract}
Torehabilitate individuals with impaired upperlimb functions we have developed an exoskeleton robot, ETS-MARSE. In this paper, we proposed and implemented a control strategy using skin surface electromyogram (EMG) signals of subjects to maneuver the developed exoskeleton robot. A nonlinear sliding mode control technique with exponential reaching law was used for this purpose where EMG signals from shoulder and elbow muscles were used as input information to the controller. To evaluate the performance of the proposed control approach experiments were carried out with the healthy subjects. Experimental results indicate that with the proposed control strategy, ETS-MARSE can be effectively maneuvered with the EMG signals both for the single and multi-joint movement assistance.
\end{abstract}

Index Terms-ETS-MARSE, electromyogram signals, nonlinear control, rehabilitation, exoskeleton

\section{INTRODUCTION}

We have been researching on the design, development and control of wearable exoskeleton robot [1], [2] for rehabilitation and assisting upper-extremity movements of individuals with impaired upper-limb function. Upperextremity impairments of the shoulder, elbow or wrist are common due to stroke, sport injuries, trauma, occupational injuries, and others. Rehabilitation programs are the main method to promote functional recovery in these individuals [3]. Research studies reveal that patients who receive robot-assisted therapy show good improvement of motor skills, when compared to conventional therapy techniques [4]-[6].

The seven degrees of freedom exoskeleton robot, ETS$M A R S E$ (Fig. 1) that we have developed is able to provide every variety of arm movements, which includes shoulder, elbow, forearm, and wrist joint motions. With different control strategies the ETS-MARSE can be used to provide passive, active, and active assisted therapy.

In our previous research [7], [8], we proposed and implemented control strategies using a computed torque control and a PID control to maneuver the ETS-MARSE in providing passive rehabilitation therapy (passive

Manuscript received April 15, 2014; revised September 17, 2014. movement tracking). In this rehabilitation approach, the subjects remain passive and cannot actively take part in the therapeutic sessions due to the severe nature of their impairments, e.g., major weakness or paralysis of the arm muscles; therefore the robot's task is to assist in performing the appropriate exercises (such as shoulder flexion/extension etc.). Following some passive therapeutic sessions and once the joints' range of motion of subjects are increased, the next approach is known as active rehabilitation therapy, where subjects actively participate in the therapeutic sessions.

As a step to provide active therapy or motion assist, in this research we developed a control strategy based on subject's skin surface electromyo graphic (EMG) signals. Note that EMG signals are biologically generated signals that reflect the user's intentions of movement [9]. Therefore, it is expected that the use of EMG signals to the robot controller will help maneuvering the robot in real time in assisting subject's motion. In this research, the EMG-based control (EBC) strategy focused on the control of the ETS-MARSE for shoulder and elbow joint motion assist. Considering the nonlinear dynamics of the ETS-MARSE and the human upper-extremity, the proposed $E B C$ was developed using nonlinear sliding mode control with exponential reaching law (SMERL) [10].

Although the primary input to the $E B C$ is the subject's skin surface EMG signals, these EMG signals are transformed to a position command, therefore the driving principle of the ETS-MARSE is a position controller based on the EMG signals. Experiments were carried out with healthy male subjects where the ETS-MARSE was maneuvered with the EMG signals. It is expected that with the proposed EBC, the ETS-MARSE can assist physically disabled individuals by providing them the motion assistance in arm movements.

In the next section of this paper, a brief overview of the ETS-MARSE is presented. Section III describes the control strategies. In section IV, experimental results are presented to evaluate the performance of the $E B C$ and the ETS-MARSE with regard to motion assist, and finally, the paper ends with the conclusion and future works in section V. 


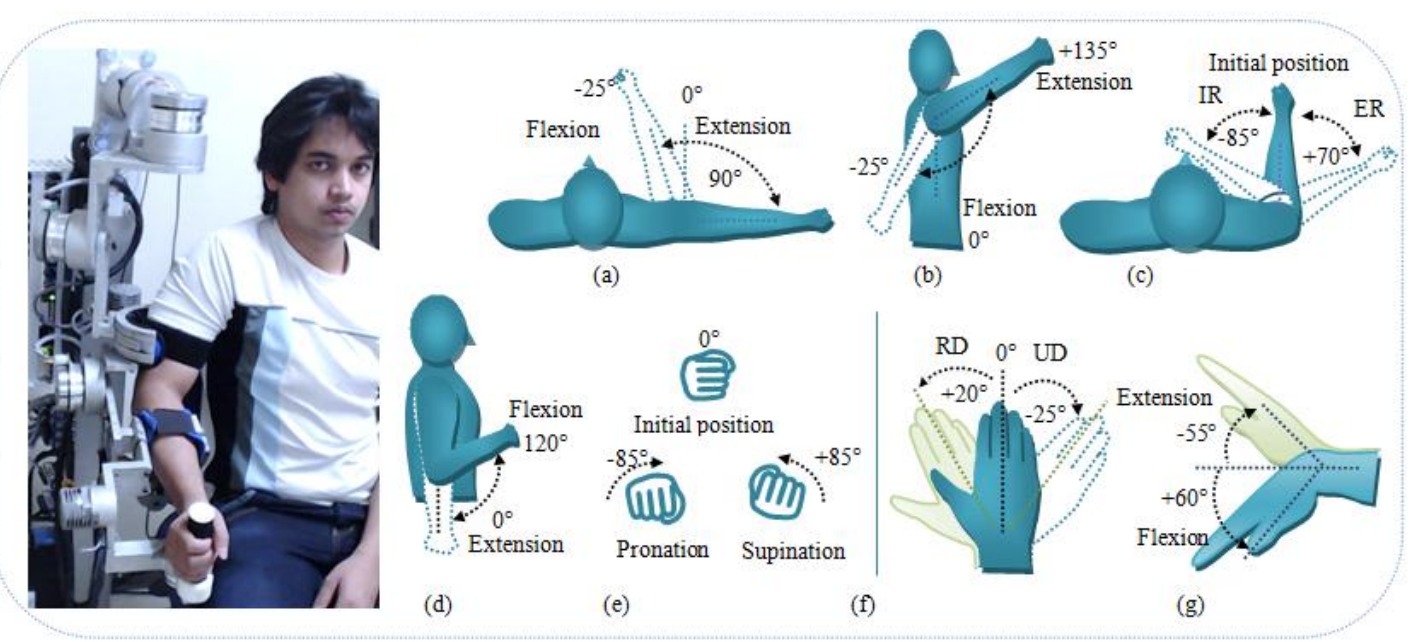

Figure 1. Exoskeleton robot, ETS-MARSE and its workspace (a) Joint-1: shoulder joint horizontal flexion-extension; (b) Joint-2: shoulder joint vertical flexion-extension; (c) Joint-3: shoulder joint internal rotation (IR)-external rotation (ER); (d) Joint-4: elbow flexion-extension; (e) Joint-5:

forearm pronation-supination; (f) Joint-6: wrist joint radial deviation (RD)-ulnar deviation (UD); (g) Joint-7: wrist joint flexion-extension.

\section{RoBotic EXOSKELETON, ETS-MARSE}

The exoskeleton robot, ETS-MARSE and its workspace are shown in Fig. 1.The robot was designed to be worn on the lateral side of upper extremity. Provision included in the design to adjust the link-lengths to accommodate a wide range of users. The robot has seven degrees of freedoms (shoulder: 3DoFs, elbow: 1DoF, forearm: 1DoF, wrist: 2DoFs). It was designed to provide naturalistic movements of the shoulder (i.e., vertical and horizontal flexion/extension, and internal/external rotation), elbow (i.e., flexion/extension), forearm (i.e., pronation/supination), and wrist joint (i.e., radial/ulnar deviation, and flexion/extension). Brushless DC motors incorporated with harmonic drives are used to actuate the ETS-MARSE. As a safety features mechanical stoppers are added at each joint to limit the joints' rotation within the range of ETS-MARSE's workspace.

\section{CONTROL}

\section{A. EMG Signals Processing and Muscle Modeling}

The EMG signals, which are widely used as a measure of muscle activity, are highly nonlinear in nature. They vary from subject to subject and also depending on the subject's physiological conditions such as fatigue, stress, placement of electrodes etc. [9] The EMG signal contains a wide range of frequencies making it difficult to reduce noise by filtering. Therefore, raw EMG signals cannot be directly used by the controller. So, features have to be extracted from the noisy raw EMG signals. Among the commonly used feature extraction methods (such as, zero crossing, waveform length, mean absolute value, root mean square, average or full rectified value, slope sign changes, etc. [11]), for this study we have used root mean square (RMS) value to process raw EMG signals $( \pm 5 \mathrm{mV})$. Note that the RMS value is a measure of power of the signal. The equation of RMS value is written as:

$$
R M S=\sqrt{\frac{1}{N} \sum_{i=1}^{N} v_{i}^{2}}
$$

where $v_{i}$ is the voltage value at the $i$ th sampling and $N$ is the number of sample in a segment. In our study, the number of samples was set to 50 and the sampling time was $0.5 \mathrm{msec}$.

An example of the RMS value of raw EMG signals recorded from shoulder muscles (anterior part of deltoid) is depicted in Fig. 2.
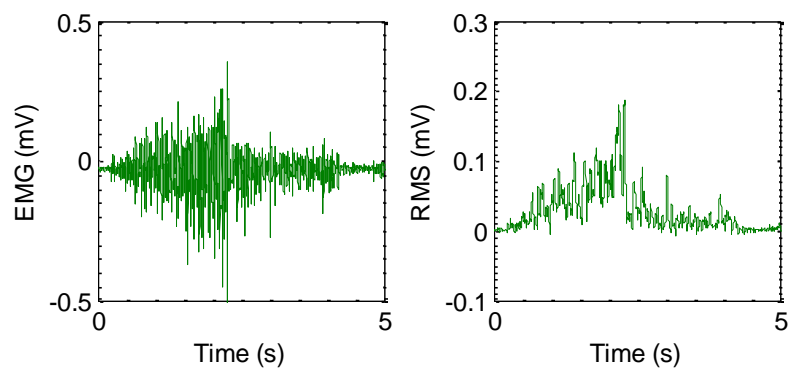

Figure 2. Example of raw EMG signals (deltoid) and RMS values

Anatomical joint movements are controlled by the actions of agonist and antagonist muscles (e.g., anterior part of deltoid for shoulder flexion, posterior part of deltoid for shoulder extension motion etc.). In some cases, for single joint movements, multiple agonists and antagonists muscles may be involved (e.g., shoulder joint vertical flexion is controlled by the posterior part of deltoid, teres major, and latissimus dorsi muscles) [12]. In this research we have studied the shoulder and elbow muscles to find an appropriate pair of agonist and antagonist muscles responsible for shoulder and elbow joint movements. Table I gives the antagonist and agonist muscles used to develop the proposed $E B C$. The study was experimentally validated by analyzing EMG activity of healthy individuals' muscles. 
TABLE I. DOMINATING AGONIST AND ANTAGONIST MuscLES OF SHOULDER AND ELBOW [12]

\begin{tabular}{|c|c|}
\hline \hline Types of motion & Agonist/Antagonist Muscles \\
\hline Shoulder joint vertical flexion & Deltoid anterior part \\
\hline Shoulder joint vertical extension & Deltoid posterior part \\
\hline Elbow flexion & Biceps brachii \\
\hline Elbow extension & Triceps brachii \\
\hline \hline
\end{tabular}

B. Control Strategy

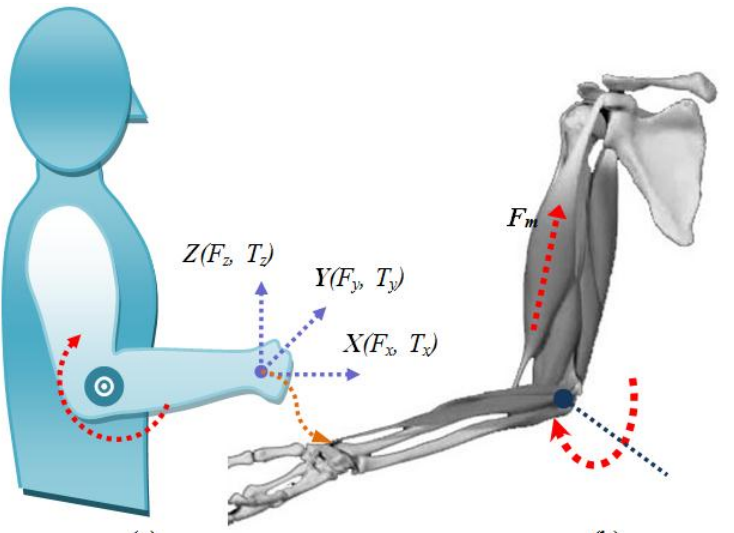

(b)

Figure 3. Elbow joint flexion/extension mechanism. (a) Virtual force acting at the end-effector (wrist-joint), producing joint torques, (b) Elbow muscles producing equivalent elbow joint torques causing flexion/ extension motion.

The skeletal muscles forces (i.e., contractions and tensions) produce necessary joint torques for posture control and voluntary movements. If a force/torque applied at the wrist joint of upper-limb (or at the endeffector of ETS-MARSE), it can produce the same joint torques, and may results the same joint motions. For instance, as shown in Fig. 3a, a force applied along $Z$ axis (i.e., $F_{z}$ ) or a torque applied around $Y$ axis (i.e., $T_{y}$ ) can produce the same torque as produced by the elbow muscles force $\left(F_{m}\right.$, Fig. $\left.3 b\right)$ at the elbow joint. Similarly a torque applied around $X$ axis $\left(T_{x}\right)$ will produce the same forearm motion as produced by the actions of pronator teres and supinator muscles. Therefore, in this case, the surface EMG signals, which are a measure of muscle activity, can be co-related to a virtual force/torque $\left(F_{v}\right)$ acting at the wrist joint of a subject. Let's assume that EMG signals recorded from the agonist and antagonist muscles $\left(E M G_{i}\right)$ for individual joint movements are proportional to the virtual force/torque vectors $\left(F_{v} \in \mathbb{R}^{6 \times 1}\right.$ ). The relation can be expressed as:

$$
F_{v}=\Delta C_{i} \times E M G_{i}
$$

where $\Delta C_{i}=$ proportional constant for individual muscles.

Therefore, the virtual force/torque vector that represents the activity of agonist and antagonist muscles of elbow would be $F_{v_{-} e l}=\left[\begin{array}{llllll}0 & 0 & F_{z} & 0 & T_{y} & 0\end{array}\right]^{T}$

where $F_{z}=\Delta C_{f_{z} i} \times E M G_{i}$, and $T_{y}=\Delta C_{T_{y} i} \times E M G_{i}$.
Similarly, for shoulder joint vertical flexion/extension motion due to the activity of agonist and antagonist muscles of shoulder, it would be $F_{v_{-} s R}=\left[\begin{array}{llllll}F_{x} & 0 & F_{z} & 0 & T_{y} & 0\end{array}\right]^{T}$.

Consider a 6-axis virtual force/torque sensor (representing EMG activity of muscles)is instrumented at the end-effector of the ETS-MARSE. The simplified control architecture of the ETS-MARSE control system is depicted in Fig. 4. The principle of $E B C$ approach (or active exercise) is that the subjects initiate the movement to complete a specified task.

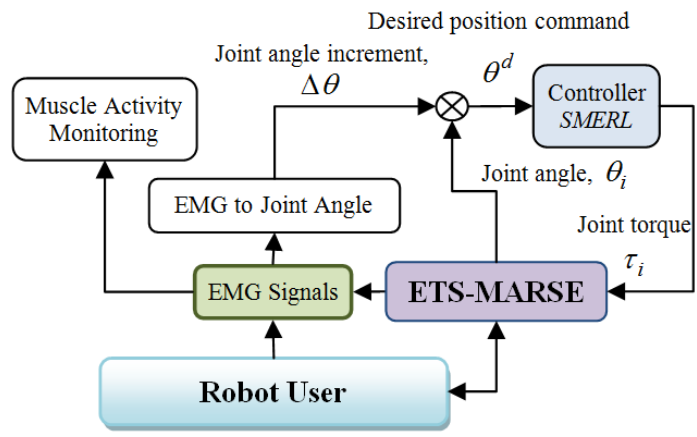

Figure 4. Schematic diagram of EMG based control system

As seen from the schematic (Fig. 4), control is carried out based on the subjects' EMG signals. The instantaneous EMG signals of muscles (i.e., subject's initiation of movement) and the previous joint angles of the ETS-MARSE are used to compute the desired position command (joint angles) of the robot to follow, which can be expressed as:

$$
\theta^{d}=\theta_{\text {old }}+\Delta \theta\left(\tau_{v}\left(E M G_{i}\right)\right)
$$

where $\theta^{d} \in \mathbb{R}^{7 \times 1}$ is the desired joint angles vector, $\theta_{\text {old }} \in \mathbb{R}^{7 \times 1}$ is the previous joint angles vector, and $\Delta \theta \in \mathbb{R}^{7 \times 1}$ is the vector of infinitesimal changes of joints angles corresponding to the instantaneous change of EMG signals, and $\tau_{v} \in \mathbb{R}^{7 \times 1}$ is the virtual torques vector. Note that, the virtual force/torques $F_{v} \in \mathbb{R}^{6 \times 1}$ as applied at the end-effector corresponding to the instantaneous change of EMG signals produce joints' torques ( $\tau_{v} \in \mathbb{R}^{7 \times 1}$ ) which can be expressed by the following relation.

$$
\tau_{v}=J^{T}(\theta) F_{v}
$$

where $J(\theta) \in \mathbb{R}^{6 \times n}$ is the Jacobian matrix of ETS-MARSE ( $n=7$ ), and $\theta \in \mathbb{R}^{7 \times 1}$ is the joint angle vector.

The change of joints' angle corresponding to theinfinitesimal changes of $F_{v} \in \mathbb{R}^{6 \times 1}$ is thus assumed to be proportional to the corresponding joints' torques $\Delta \tau_{v} \in \mathbb{R}^{7 \times 1}$, that is-

$$
\Delta \tau_{v}=K_{p G} \Delta \theta
$$


where $K_{p G} \in \mathbb{R}^{7 \times 7}$ is the (diagonal) proportional gain matrix that transforms infinitesimal changes of joints' torques $\left(\Delta \tau_{v}\right)$ to the corresponding joint angles $(\Delta \theta)$. A detail of this transformation can be found in [8].

So, the sliding mode exponential reaching law (SMERL), as depicted in the schematic (Fig. 5), works mainly on the position regulation of the ETS-MARSE. In the next sub-section, the theoretical structure of the sliding mode control technique with exponential reaching law (ERL) is presented for the dynamic trajectory tracking of the ETS-MARSE.

\section{Sliding Mode Control with ERL}

The dynamic behaviour of the ETS-MARSE can be expressed by the rigid body dynamic equation as:

$$
M(\theta) \ddot{\theta}+V(\theta, \dot{\theta})+G(\theta)+F(\theta, \dot{\theta})=\tau
$$

where $M(\theta) \in \mathbb{R}^{7 \times 7}$ is the inertia matrix, $V(\theta, \dot{\theta}) \in \mathbb{R}^{7 \times 1}$ is the coriolis/centrifugal vector, $G(\theta) \in \mathbb{R}^{7 \times 1}$ is the gravity vector, and $F(\theta, \dot{\theta}) \in \mathbb{R}^{7 \times 1}$ is the friction vector which is modeled as a nonlinear coulomb friction, and can be expressed as:

$$
\tau_{\text {friction }}=F(\theta, \dot{\theta})=f_{c} 3 g n(\dot{\theta})
$$

where $f_{c}$ is the coulomb-friction constant. Equation (6) can be written as:

$$
\begin{gathered}
\ddot{\theta}=-M^{-1}(\theta)\left[V(\theta, \dot{\theta})+G(\theta)+f_{c} \xi g n(\dot{\theta})\right] \\
+M^{-1}(\theta) \tau
\end{gathered}
$$

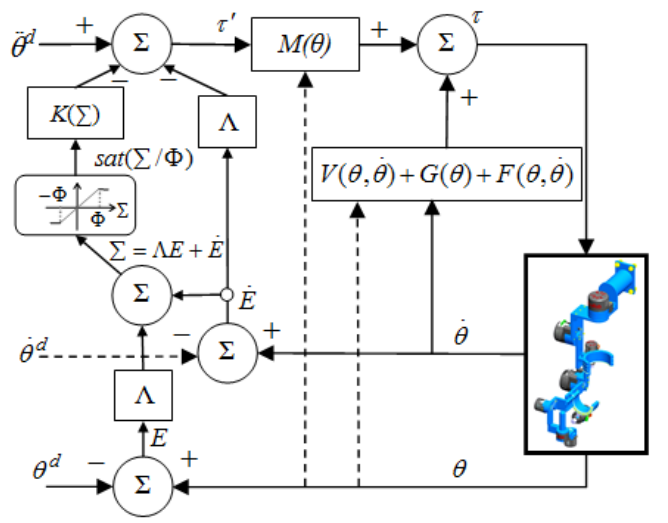

Figure 5. Schematic diagram of sliding mode exponential reaching law in combination with sat function

The general layout of the SMERL technique is depicted in Fig. 5. Let the tracking error for each joint be defined as:

$$
e_{i}=\theta_{i}-\theta_{i}^{d}(i=1, ? \cdot \cdot m)
$$

And the sliding surface $(S)$ as:

$$
S_{i}=\lambda_{i} e_{i}+\dot{e}_{i} \quad(i=1, ? \cdot \cdot m)
$$

where $\theta_{i}^{d}$ is the desired trajectory for joint $i$ and $S_{i}$ is the sliding surface of each DoF.

Let $\Sigma=\left[\begin{array}{llll}S_{1} & S_{2} & \cdots & S_{m}\end{array}\right]^{T}$ be the sliding surface for the ETS-MARSE. Considering the following nonnegative and continuous Lyapunov function candidate:

$$
V=\frac{1}{2} \Sigma^{T} \Sigma
$$

The derivative of $V$ yields:

$$
\dot{V}=\Sigma^{T} \dot{\Sigma}
$$

By choosing $\dot{\Sigma}$ as given in equation (13) relation (12) is ensured to be decreasing.

$$
\dot{\Sigma}=-K(\Sigma) \operatorname{sat}(\Sigma / \phi), \forall t, K(\Sigma)>0 \Rightarrow \dot{V}<0
$$

where

$$
\operatorname{sat}(\Sigma / \phi)=\left\{\begin{array}{ccc}
\Sigma / \phi & \text { for } & |\Sigma / \phi| \leq 1 \\
0 & \text { for } & \Sigma / \phi \\
& & \\
\operatorname{sign}(\Sigma / \phi) & \text { for } & |\Sigma / \phi|>1
\end{array}\right.
$$

Expression (13) is known as the reaching law that combines the concept of ERL [10] with sat function [13]in order to reduce chattering in control signals and improve tracking both in transient and steady state condition. Note that, the convergence of the system stays within a boundary layer neighborhood (as defined by $\Phi$ ) of the switching surface. More details on the development of this reaching law can be found in [14]. Now considering,

$$
\begin{gathered}
\dot{E}=\left[\begin{array}{llll}
\dot{e}_{1} & \dot{e}_{2} & \cdots & \dot{e}_{m}
\end{array}\right]^{T}, \text { and } \Lambda=\operatorname{diag}\left[\begin{array}{lll}
\lambda_{i} & \cdots & \lambda_{m}
\end{array}\right] \\
\Sigma=\Lambda E+\dot{E} \Rightarrow \quad \Rightarrow \quad \dot{\Sigma}=\Lambda \dot{E}+\ddot{E}
\end{gathered}
$$

where, $\ddot{E}=\ddot{\theta}-\ddot{\theta}^{d}$.Thus, relation (14) can be written as:

$$
\dot{\Sigma}=\Lambda \dot{E}+\ddot{\theta}-\ddot{\theta}^{d}
$$

Substituting the value of $\ddot{\theta}$ from equation (8) in equation (15) we obtain:

$$
\begin{aligned}
\dot{\Sigma} & =\Lambda \dot{E}-\ddot{\theta}^{d}+M^{-1}(\theta) \tau \\
& -M^{-1}(\theta)\left[V(\theta, \dot{\theta})+G(\theta)+f_{c} 3 g n(\dot{\theta})\right]
\end{aligned}
$$

Replacing $\dot{\Sigma}$ by its value given in equation (13):

$$
\begin{gathered}
-K(\Sigma) \operatorname{sat}(\Sigma / \phi)=\Lambda \dot{E}-\ddot{\theta}^{d} \\
-M^{-1}(\theta)\left[V(\theta, \dot{\theta})+G(\theta)+f_{c} 3 g n(\dot{\theta})-\tau\right]
\end{gathered}
$$

The torque $\tau$ can be isolated and thus yields:

$$
\begin{aligned}
\tau & =-M(\theta)\left(\Lambda \dot{E}-\ddot{\theta}^{d}+K(\Sigma) \operatorname{sat}(\Sigma / \phi)\right) \\
& +\left[V(\theta, \dot{\theta})+G(\theta)+f_{c} 3 g n(\dot{\theta})\right]
\end{aligned}
$$

where $K$ and $\Lambda$ are diagonal positive definite matrices. Therefore, the control law given in equation (18) ensures 
that the control system is stable.

\section{EXPERIMENTS AND RESULTS}

Experimental setup of the ETS-MARSE system is depicted in Fig. 6. It consists of a CPU processor (NI PXI-8108) with a reconfigurable FPGA (fieldprogrammable gate array), seven motor drivers, a custom designed main board, and a host PC. The main board as shown in Fig. 6 acts as a motherboard which routes various analog and digital signals from/to the NI-PXI to/from the ETS-MARSE system. The control algorithm is executed in the NI-PXI.

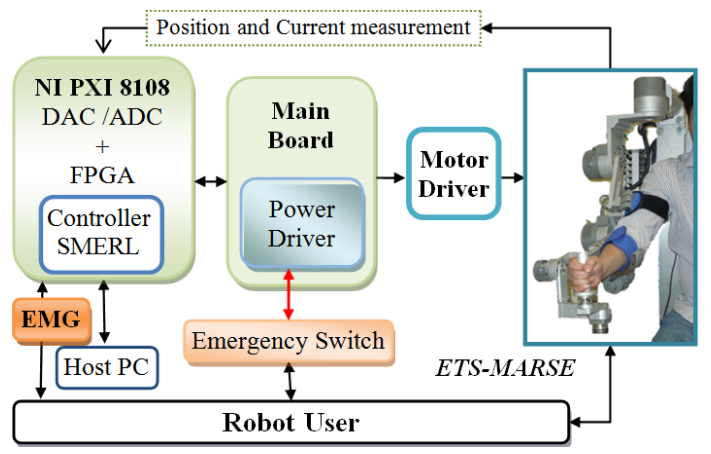

Figure 6. Experimental setup.

The EMG electrodes (Delsys) placement on upperlimb muscles are depicted in Fig. 7. In this study, two elbow muscles (biceps and triceps), and two shoulder muscles (deltoid: anterior and posterior parts) of the subjects were monitored and used as an input information to control of the ETS-MARSE. In experiments, the performance of the proposed $E B C$ approach for motion control of the ETS-MARSE was evaluated. Note that, the experiments were conducted with subjects in a seated position with elbow joint angle at $90^{\circ}$.

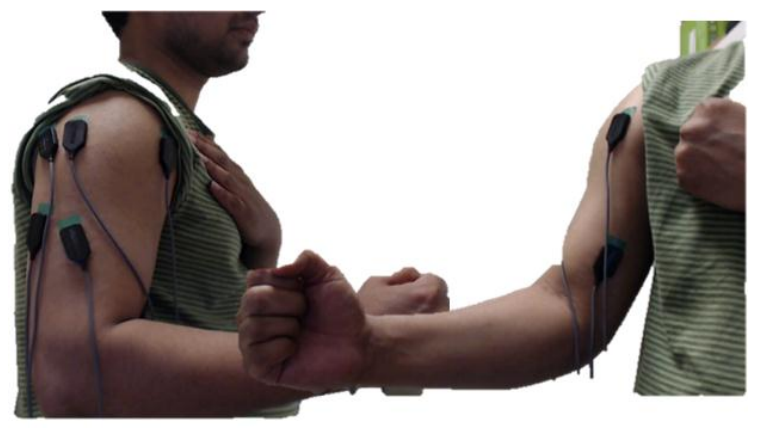

Figure 7. Electrode placements (shoulder and elbow)

The experimental results for elbow joint flexion/extension movement are depicted in Fig. 8. The topmost plots shows the RMS values of EMG signals recorded from the biceps brachii muscle and that for the triceps is shown in middle. The elbow joint angles of ETS-MARSE corresponding to the biceps and triceps actions are shown in the bottom of Fig. 8. It can be seen from Fig. 8, that when triceps is activating (e.g., at $t=0.5 \mathrm{~s}$, $3.5 \mathrm{~s}, 6.7 \mathrm{~s}, 9 \mathrm{~s})$ it produces extension motion of ETS$M A R S E$ and when biceps is activating (at $t=1.8 \mathrm{~s}, 5.3 \mathrm{~s}$, $7.8 \mathrm{~s}, 9.7 \mathrm{~s})$ it produces flexion motion of ETS-MARSE.
From Fig. 8, it can also be found that when there was no muscle activity such as at $t=2.5 \mathrm{~s}$ to $3.5 \mathrm{~s}$, the ETS-MARSE stayed at the same position and was not affected due to gravity.
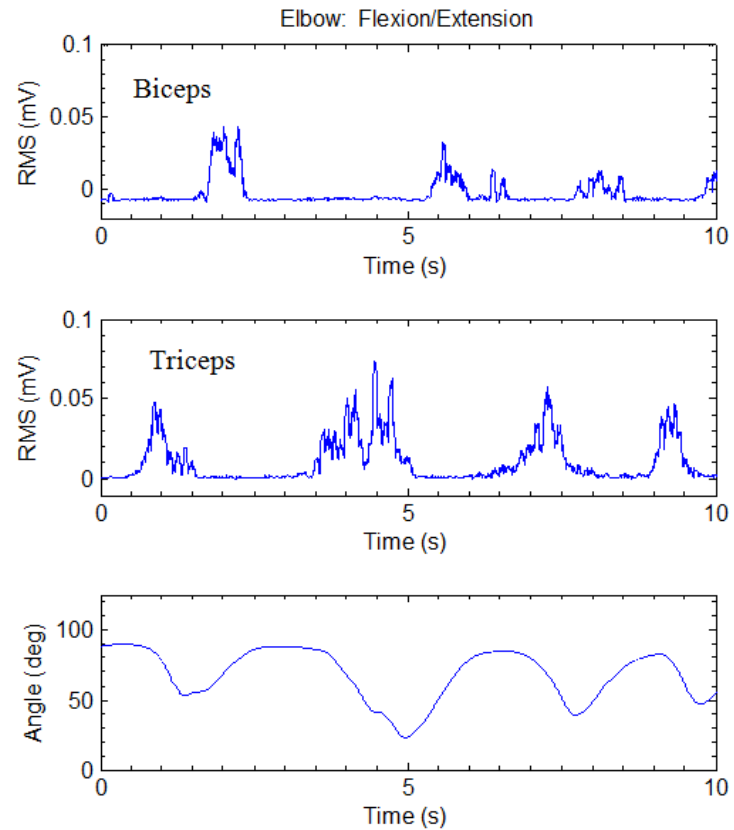

Figure 8. Elbow joint flexion/extension
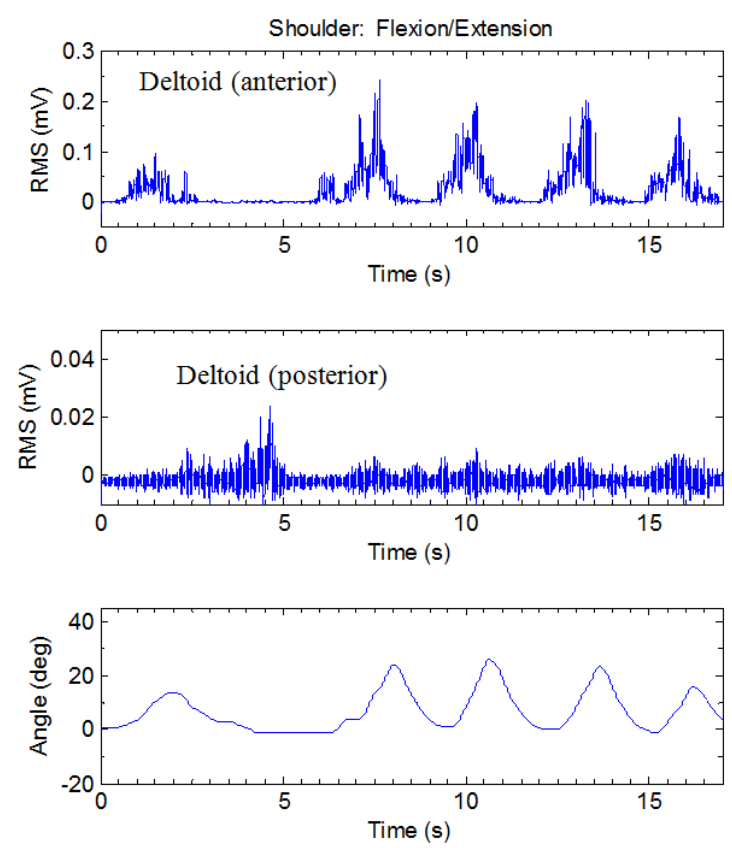

Figure 9. Shoulder joint flexion/extension motion

The experimental results for shoulder joint vertical flexion/extension movement are depicted in Fig. 9, where it can be seen that the anterior part of the deltoid is responsible for the vertical flexion motion of the ETSMARSE. Mechanical stoppers were used in this case to limit the shoulder joint extension motion to zero degree. Therefore, it can be also seen from Fig. 9 that the posterior part of the deltoid producing the extension motion of the ETS-MARSE and limits the motion to $0^{\circ}$. 

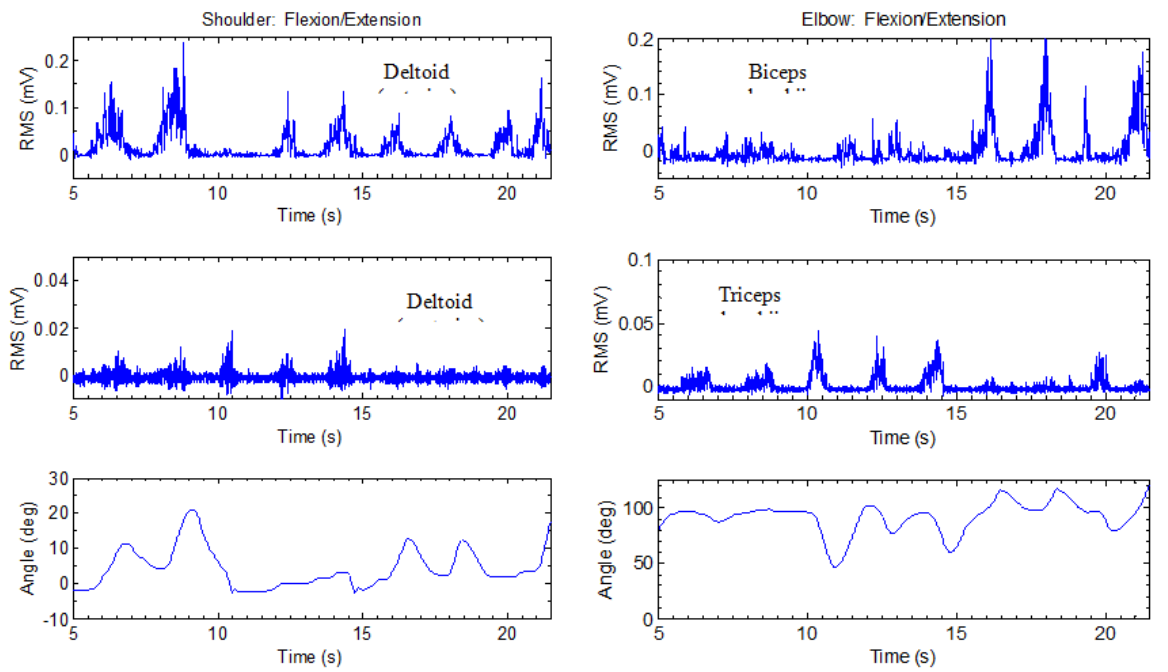

(a)

(b)

Figure 10. Simultaneous movements of shoulder and elbow (reaching movements)

To further evaluate the performance of $E B C$, a multijoint movement exercise such as simultaneous movements of shoulder and elbow joints (i.e., reaching movements) was performed. The experimental results are depicted in Fig. 10. It can be seen from the plots that the exercise began with shoulder joint flexion/extension motions (Fig. 10a) and continue up to $t=11 \mathrm{~s}$, then elbow joint flexion/extension motions were performed up to $t=14 \mathrm{~s}$, and finally simultaneous motions of elbow and shoulder joints were performed for the rest of trial. Note that the trial was performed in different velocities for $22 \mathrm{~s}$. In all cases the developed $E B C$ was able to maneuver the ETS-MARSE effectively in providing shoulder and elbow joint motions using the EMG signals of muscles. Therefore, the control approach as presented in this research can be effectively used in providing active rehabilitation exercises.

\section{CONCLUSION}

To reflect user intention of motion, surface EMG signals were used to develop a control strategy for the motion of an exoskeleton robot, named ETS-MARSE. The performance of the controller was experimentally evaluated for single and multi-joint motion control of the robot. Experimental results confirmed that with the developed control approach the ETS-MARSE will be able to provide active assistance in arm movements. Future work will include developing a hybrid controller using fusion of the EMG and force sensor signals.

\section{ACKNOWLEDGMENT}

The first author gratefully acknowledges the support provided for this research through a FRQNT-B3 fellowship. The authors also acknowledge the support provided for this research by the INTER research group.

\section{REFERENCES}

[1] M. H. Rahman, M. J. Rahman, O. L. Cristobal, M. Saad, J. P. Kenne, and P. S. Archambault, "Development of a whole arm wearable robotic exoskeleton for rehabilitation and to assist upper limb movements," Robotica, pp. 1-21, 2014.

[2] M. H. Rahman, T. K. Ouimet, M. Saad, J. P. Kenne, and P. S. Archambault, "Development and control of a robotic exoskeleton for shoulder, elbow and forearm movement assistance," Applied Bionics and Biomechanics, vol. 9, no. 3, pp. 275-292, 2012.

[3] G. E. Gresham, D. Alexander, D. S. Bishop, C. Giuliani, G. Goldberg, A. Holland, M. Kelly-Hayes, R. T. Linn, E. J. Roth, W. B. Stason, and C. A. Trombly, "American heart association prevention conference. IV. prevention and rehabilitation of stroke. rehabilitation," Stroke, vol. 28, no. 7, pp. 1522-1526, July 1997.

[4] P. S. Lum, C. G. Burgar, P. C. Shor, M. Majmundar, and M. Van der Loos, "Robot-assisted movement training compared with conventional therapy techniques for the rehabilitation of upperlimb motor function after stroke," Archives of Physical Medicine and Rehabilitation, vol. 83, no. 7, pp. 952-959, 2002.

[5] S. Masiero, A. Celia, G. Rosati, and M. Armani, "Robotic-assisted rehabilitation of the upper limb after acute stroke," Arch Phys Med Rehabil, vol. 88, no. 2, pp. 142-149, Feb. 2007.

[6] N. N. Gheidari, P. Archambault, and J. Fung, "Effects of robotassisted therapy on stroke rehabilitation in upper limbs: systematic review and meta-analysis of the literature," J Rehabil Res Dev., vol. 49, no. 4, pp. 479-96, 2012.

[7] M. H. Rahman, M. Saad, J. P. Kenne, and P. S. Archambault, "Robot assisted rehabilitation for elbow and forearm movements," Int. J. Biomechatronics and Biomedical Robotics, vol. 1, no. 4, pp. 206-218, 2011.

[8] M. H. Rahman, T. K. Ouimet, M. Saad, J. P. Kenne, and P. S. Archambault, "Development of a 4DoFs exoskeleton robot for passive arm movement assistance," Int. J. Mechatronics and Automation, vol. 2, no. 1, pp. 34-50, 2012.

[9] K. Kiguchi, M. H. Rahman, and T. Yamaguchi, "Adaptation strategy for the 3DOF exoskeleton for upper-limb motion assist," in Proc. 2005 IEEE International Conference on Robotics and Automation, Barcelona, Spain, 2005, pp. 2296-2301.

[10] C. J. Fallaha, M. Saad, H. Y. Kanaan, and K. Al-Haddad, "Slidingmode robot control with exponential reaching law," IEEE Transactions on Industrial Electronics, vol. 58, no. 2, pp. 600-610, 2011.

[11] K. Kiguchi, M. H. Rahman, M. Sasaki, and K. Teramoto, "Development of a 3DOF mobile exoskeleton robot for human upper-limb motion assist," Robotics and Autonomous Systems, vol. 56, no. 8, pp. 678-691, Aug 31, 2008.

[12] H. Gray and C. D. Clemente, Anatomy of the Human Body, 30th American ed. Philadelphia: Lea \& Febiger, 1985.

[13] H. Khalil, Nonlinear Systems, 3rd ed. Upper Saddle River, N.J.: Prentice Hall, 2002.

[14] M. H. Rahman, M. Saad, J. P. Kenne, and P. S. Archambault, "Control of an exoskeleton robot arm with sliding mode exponential reaching law," International Journal of Control, Automation and Systems, vol. 11, no. 1, pp. 92-104, 2013. 


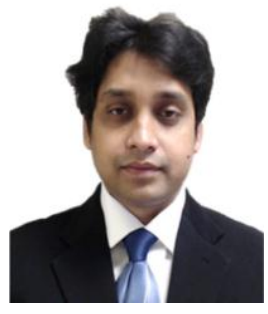

Mohammad Habibur Rahmanis a postdoctoral research fellow with the School of Physical \& Occupational Therapy, McGill University, and a researcher in the biorobotics research lab at the École de technologiesupérieure, Montreal, Canada. $\mathrm{He}$ received BSc engineering (mechanical) from Khulna University of Engineering \& Technology (KUET), Khulna, Bangladesh in 2001, and Master of Engineering (mechanical-biorobotics) from Saga University, Japan in 2005. In 2012, he received a PhD in engineering (bio-robotics) from École de technologiesupérieure, Université du Québec.He joined as a $\mathrm{L}$ ecturer in the mechanical engineering department, KUET, Bangladesh in 2001 and later served as an Assistant Professor in the same department from 2006 to 2012. His research interests are in rehabilitation robotics, wearable robots, intelligent systems and control, tele-operation, and nonlinear control.Email: mhrahman@ieee.org.

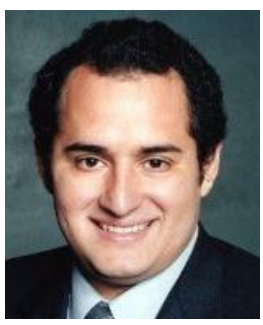

Cristóbal Ochoa Lunais (Ph. D candidate) with the electrical engineering department (specialization: robotics), École de technologiesupérieure (ETS) in Montreal, Quebec, Canada. He studies under National Council on Science and Technologie of Mexico (CONACYT)-ETS scholarship. He received a MSc in Electronics Engineering and a Bachelor in Electronic and Computer Engineering from the University of the Americas Puebla in 2003 and 2005 respectively. He worked in 'Engineering Automation, Control and Communications' in Puebla, February 2006 to August 2009 as a Project Engineer, performing functions of project integration and industrial control automation, consulting, improvement, maintenance and troubleshooting in production processes. It originates from the port of Acapulco, Guerrero, Mexico. His areas of interest are the control and robotics. Email: cristobal.ochoa-luna.1@ens.etsmtl.ca.

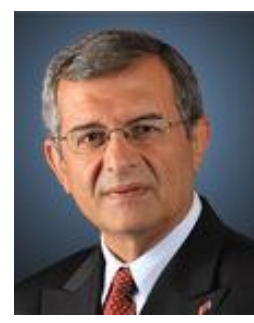

Maarouf Saadis a professor in the department of electrical engineering of École de technologiesupérieure, Université du Québec, Montreal, Canada. He received a bachelor and a master degree in electrical engineering from Ecole Polytechnique of Montreal respectively in 1982 and 1984. In 1988, he received a Ph.D from McGill University in electrical engineering. He joined École de technologiesupérieure in 1987 where he is teaching control theory and robotics courses. His research interests are in nonlinear control and optimization applied to robotics and fligh control system, rehabilitation robotics, power systems and distributed generation.Email: maarouf.saad@etsmtl.ca.

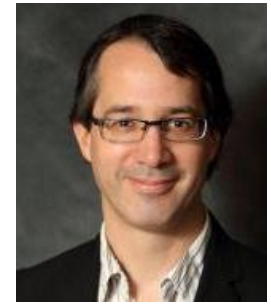

Philippe Archambaultis an Associate Professor at the School of Physical and Occupational Therapy, McGill University, and a researcher at the Interdisciplinary Research Center in Rehabilitation (CRIR), Montreal, Canada. He received his undergraduate degrees in Physics and Occupational Therapy from McGill University. He received a MSc in Biomedical Engineering from University de Montréal, and a $\mathrm{PhD}$ in neuroscience, from the same university. His research interests are in motor control, technology in rehabilitation, robotics, wheelchair mobility, and virtual reality. He is teaching neurophysiology and assistive technology. Email philippe.archambault@mcgill.ca. 